\title{
On Multipath Clustering in ANGUlar Domain AND ITS IMPACT ON ChaNNEL CAPACITY AND DIVERSITY GAIN
}

\author{
Guangze Zhao, Sergey Loyka \\ School of Information Technology and Engineering (SITE) \\ University of Ottawa, 161 Louis Pasteur, Ottawa, Ontario, K1N 6N5, Canada \\ sloyka@site.uottawa.ca
}

\begin{abstract}
The effect of multipath clustering in angular domain and its impact on multi-antenna (MIMO) system performance (capacity, diversity gain) is studied in details. Fourier transform technique is a powerful tool that provides insight into the relationship between the angular spectrum (or angular probability density function(PDF)) of the incoming multipath and the antennas' signal correlation. Compact closed-form expressions are derived and validated in the case of small angular spread in each cluster. Optimization problem is studied in terms of correlation and capacity/diversity gain. It is demonstrated that the capacity/diversity gain can be maximized by the antenna array orientation in such a way that individual cluster contributions to the correlations add in a destructive way. A new way to determine the angular PDF from measured data is suggested.
\end{abstract}

Index Terms-Multipath propagation, fading correlation, MIMO system performance

\section{INTRODUCTION}

The high-performance promise of multi-antenna (MIMO) systems can be significantly deteriorated by subchannel correlation of the MIMO channel. Careful modeling of correlation and its impact on the MIMO system performance is, hence, of high importance $[1,3,4]$. Since the angular spectrum of the incoming multipath has ultimately related to the correlation and can also be measured for practical channels, its study attracted significant attention in recent years [2-7]. Most works are concentrated on a single-cluster channel with various angular probability density functions (PDF) or power spectra [2-4]. Considerably less attention is paid to multicluster channels. Extensive measurements of practical channels indicate, however, that multipath often arrives in several clusters in angular domain (in addition to its cluster in delay time domain), which was observed for both indoor and outdoor channels. Geometrical considerations can provide a straightforward explanation for this.

Hence, we concentrate out attention in the present paper on multipath angular clustering and its impact on correlation and capacity/diversity gain. Wherever possible, analytical techniques are used since they provide considerable insight (even when approximations are involved), which is not attainable using numerical methods. Multiple clusters add new dimension to the system optimization problem, allowing to achieve lower correlation and, hence, higher capacity and diversity gain by adjusting both the antenna element spacing and its orientation. This optimization problem is discussed in details. While compact closed-form solutions can be obtained for 2 and 3-antenna systems, more complex ones require for numerical analysis. In most cases, however, the conclusions are still the same as for simpler 2 and 3antenna systems.

\section{ANGULAR SPECTRUM AND CORRELATION}

In this section, we briefly review the relationship between angular spectrum of the incoming multipath and antenna signal correlation [7] and demonstrate that Fourier transform (FT) is a powerful tool, which provides significant insight into the problem in general case, without resorting to specific angular spectrum models, especially in the case of small angular spread for each cluster. For simplicity, let us consider 2-D scenario (all multipath is located in a horizontal plane) as in Fig 1, assuming negligible delay spread, static channel and no polarization-specific effects, i.e. we study angular spectrum related effects only. Using the plane-wave spectrum $F\left(k_{x}\right)$ of incoming multipath, the signal along OX line can be found as,

$$
s(x)=\frac{1}{2 \pi} \int_{-k}^{k} F(k x) e^{j k_{x} x} d k_{x}
$$

where $k=2 \pi / \lambda$ is the wave vector magnitude, $\lambda$ is the wavelength, $k_{x}=k \sin \theta$ is the $\mathrm{x}$-component of the wave vector, $\theta$ is the angle of arrival (AOA) measured from the broadside (orthogonal to $\mathrm{OX}$ ). When two antennas are located along $\mathrm{OX}$ at $x_{1}$ and $x_{2}$, the signal correlation is

$$
\begin{aligned}
& R=\left\langle s\left(x_{1}\right) s^{*}\left(x_{2}\right)\right\rangle \\
& =\frac{1}{4 \pi^{2}} \iint\left\langle F\left(k_{x_{1}}\right) F^{*}\left(k_{x_{2}}\right)\right\rangle e^{j\left(k_{x_{1}} x_{1}-k_{x_{2}} x_{2}\right)} d k_{x_{1}} d k_{x_{2}}
\end{aligned}
$$

Assuming uncorrelated scattering, $\left\langle F\left(k_{x_{1}}\right) F^{*}\left(k_{x_{2}}\right)\right\rangle=2 \pi \delta\left(k_{x_{1}}-k_{x_{2}}\right) P\left(k_{x_{1}}\right)$, where $\delta(x)$ is Dirac delta function, and $P\left(k_{x}\right)$ is the angular power spectrum, (2) reduces to

$$
R(\Delta x)=\frac{1}{2 \pi} \int_{-k}^{k} P\left(k_{x}\right) e^{j k_{x} \Delta x} d k_{x}
$$

where $\Delta x=x_{1}-x_{2}$. The inverse relationship is 


$$
P\left(k_{x}\right)=\int_{-\infty}^{\infty} R(\Delta x) e^{-j k_{x} \Delta x} d \Delta x
$$

Clearly, the correlation and the angular power spectrum is the Fourier transform pair, $R(\Delta x)=\operatorname{IFT}\left\{P\left(k_{x}\right)\right\}$, $P\left(k_{x}\right)=F T\{R(\Delta x)\}$. Using AOA probability density function (pdf) $f(\theta),(3)$ can be alternatively expressed as [1]

$$
R(\Delta x)=\int_{-\pi}^{\pi} f(\theta) e^{j k \sin \theta \Delta x} d \theta
$$

The AOA pdf is the angular power spectrum are related as

$$
\begin{array}{r}
f(\theta)=\frac{k}{2 \pi}|\cos \theta| P(k \sin \theta), \\
P\left(k_{x}\right)=\frac{2 \pi}{\sqrt{k^{2}-k_{x}^{2}}} f\left[\sin ^{-1}\left(\frac{k_{x}}{k}\right)\right]
\end{array}
$$

Additionally, we note that $R(\Delta x)=R^{*}(-\Delta x)$. Hence, (4) can be reduced to

$$
P\left(k_{x}\right)=2 \int_{0}^{\infty} \operatorname{Re}\left\{R(\Delta x) e^{-j k_{x} \Delta x}\right\} d \Delta x
$$

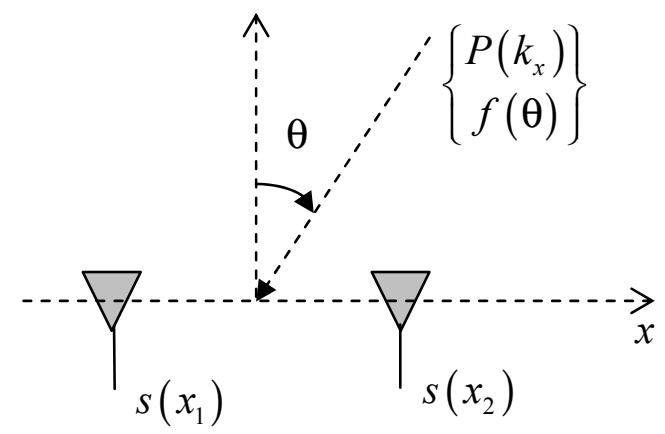

Fig 1. Illustration of the problem geometry

Let us now consider the case when the incoming multipath is concentrated within a single cluster around the broadside direction with the AOA PDF $f_{1}(\theta)$ ( $f_{1}(\theta)=0$ if $\theta \notin[-\Delta / 2, \Delta / 2], \Delta$ is the cluster width). The corresponding correlation $R_{1}(\Delta x)$ can be obtained using (5). Consider the same single cluster, which is now concentrated about mean AOA $\theta_{0}$; its AOA PDF is $f(\theta)=f_{1}\left(\theta-\theta_{0}\right)$, and its angular power density can be expressed as

$$
P\left(k_{x}\right)=f_{1}\left[\sin ^{-1}\left(k_{x} / k\right)-\theta_{0}\right] / \sqrt{k^{2}-k_{x}^{2}}
$$

Of particular interest is the case when the cluster width is small, $\Delta<<1$ (in this case, large antenna spacing is required to provide low correlation and, hence, detailed analysis is important to study possible trade-offs). Some studies using specific models of the AOA PDF for this scenario have been reported in [4,5]. Using the Fourier transform technique above, one can relate the correlation of the tilted cluster $\left(\theta_{0} \neq 0\right)$ to that of the broadside cluster $\left(\theta_{0}=0\right)$ for $\Delta<<1$ as follows. Using $\sin ^{-1}\left(k_{x} / k\right) \approx \theta_{0}+\left(k_{x}-k \sin \theta_{0}\right) / \sqrt{k^{2}-k_{x}^{2}},(8)$ reduces to

$$
P\left(k_{x}\right) \approx f_{\beta}\left[\left(k_{x}-k \sin \theta_{0}\right) / k \cos \theta_{0}\right] / k \cos \theta_{0}
$$

where we used $\sqrt{k^{2}-k_{x}^{2}}=k \cos \theta \approx k \cos \theta_{0}$. Hence, $R(\Delta x)$ can be expressed as

$$
R(\Delta x)=\operatorname{IFT}\left[P\left(k_{x}\right)\right] \approx e^{-j \Delta x \sin \theta_{0}} R_{1}\left(\Delta x \cos \theta_{0}\right)
$$

This clearly illustrates the effect of nonzero $\theta_{0}$ on the correlation. For example, larger spacing is required to achieve low correlation. Note that this relationship holds true for all AOA PDF provided that $\Delta<<1$. As extensive numerical analysis shows, it is also true for more relaxed condition of small angular spread,

$$
\sigma_{\theta}=\left(\int_{-\pi}^{\pi}\left(\theta-\theta_{0}\right)^{2} f_{\theta}(\theta) d \theta\right)^{\frac{1}{2}}<<1
$$

\section{ReCOnStruction Of THE ANGUlar POWER SPECTRUM FROM MEASURED CORRELATION}

In most measurement campaigns, the angular power spectrum (or, equivalently, AOA PDF) is measured directly using directive antennas. The analysis above suggests another way of finding the angular spectrum, using the measured correlation $R(\Delta x)$ and (7). All practical measurements, however, will determine $R(\Delta x)$ for $\Delta x \leq x_{\max }$. Hence, the integral in (7) has to be truncated,

$$
P_{t}\left(k_{x}\right)=2 \int_{0}^{x_{\max }} \operatorname{Re}\left\{R(\Delta x) e^{-j k_{x} \Delta x}\right\} d \Delta x
$$

The mean square error (MSE) of the reconstruction (due to truncation) is

$$
\varepsilon_{k}=\int_{-k}^{k}\left[P_{t}\left(k_{x}\right)-P\left(k_{x}\right)\right]^{2} d k_{x}
$$

Alternatively, one can define the reconstruction error in terms of $f(\theta)$ :

$$
\varepsilon_{\theta}=\int_{-\pi}^{\pi}\left[f_{t}(\theta)-f(\theta)\right]^{2} d \theta
$$

Using (9), they are related as

$$
\varepsilon_{\theta} \approx k \varepsilon_{k} \cos \theta_{0}
$$

Using the Parseval theorem, it can be further shown that

$$
\varepsilon_{k}=\int_{-\infty}^{\infty}\left[R_{t}(\Delta x)-R(\Delta x)\right]^{2} d \Delta x=2 \int_{x_{\max }}^{\infty}|R(\Delta x)|^{2} d \Delta x
$$

where $R_{t}$ is truncated $R$. Remarkably, the reconstruction error is related to the "tail" behavior of $R(\Delta x)$ : the larger $x_{\max }$, the smaller the error; it is also smaller when $R(\Delta x)$ decays fast. The normalized reconstruction error should be considered as more informative measure, 


$$
\varepsilon_{n}=\frac{\varepsilon\left(x_{\max }\right)}{\varepsilon(0)}
$$

As an example, we consider Gaussian and uniform AOA PDFs with $\theta_{0}=30^{\circ}, \sigma=2.88^{\circ}\left(\Delta=10^{\circ}\right) . R(\Delta x)$ for this case was reported [6]. Fig. 2 shows the reconstruction errors versus $x_{\max }$. The error is smaller for the Gaussian PDF (especially for large $x_{\max }$ ), which can be explained by faster decay of the $R(\Delta x)$ "tail" for the Gaussian PDF (exponential) as compared to uniform one (inverse linear, which is much slower due to the jumps of the PDF at the edges). The errors are almost the same for $x_{\max }<5 \lambda$. This can be explained by the fact that most contribution to the integral in (16) comes from the region of large $R$, and in that region these PDFs result in roughly the same correlation [5]. As an example, if $1 \%$ reconstruction error is desired, then $x_{\max }=8 \lambda$ and $x_{\max }=30 \lambda$ for the Gaussian and uniform PDF respectively. Fig 3 shows the recovered PDFs for $x_{\max }=16 \lambda$. Clearly, the Gaussian PDF experiences smaller reconstruction error. Since this is a consequence of the generic property of the Fourier transform (smoothly varying signal results in fastdecaying spectrum), one may expect it to be true for other AOA PDFs as well.

\section{Multi-Cluster ChanNels}

The results of Section II can be easily extended to the case of multiple clusters. We present the AOA PDF in the following form,

$$
f(\theta)=\sum_{i=1}^{N} w_{i} f_{i}\left(\theta-\theta_{i}\right)
$$

where $f_{i}(\theta)$ is the $\mathrm{i}$-th cluster AOA PDF centered around broadside, $\theta_{i}$ is its mean $\mathrm{AOA}$, and $w_{i}$ is the normalized power of i-th cluster. Using (9), the angular power density is

$$
P\left(k_{x}\right) \approx \sum_{i=1}^{N} \frac{w_{i}}{k\left|\cos \theta_{i}\right|} f_{i}\left(\frac{k_{x}-k \sin \theta_{i}}{k \cos \theta_{i}}\right)
$$

Using the linearity of the Fourier transform,

$$
R(\Delta x)=\sum_{i=1}^{N} w_{i} R_{i}(\Delta x)
$$

where $R_{i}(\Delta x)=e^{-j k x \sin \theta_{i}} R_{0 i}\left(\Delta x \cos \theta_{i}\right)$ is the $\mathrm{i}$-th cluster correlation, and $R_{0 i}$ is the correlation of $\mathrm{i}$-th cluster centered around the broadside. Clearly, the contribution of each cluster depends on its power, AOA PDF and the mean AOA. Given the same PDFs and powers, clusters deviated from the broadside contribute more to the correlation and also require more antenna spacing to reduce the correlation to a given low level. It is also clear that the clusters with small power has negligible effect on the total correlation, as it should be. For equal powers, clusters with smaller angular spread contribute most to the correlation. All these observations indicate that in a practical scenario there will be a few dominant clusters, which determine the optimum antenna design, and the other clusters can be neglected. It should be noted that for two clusters with the same PDF the results above reduce to well-known results $[5,6]$, as it should be.

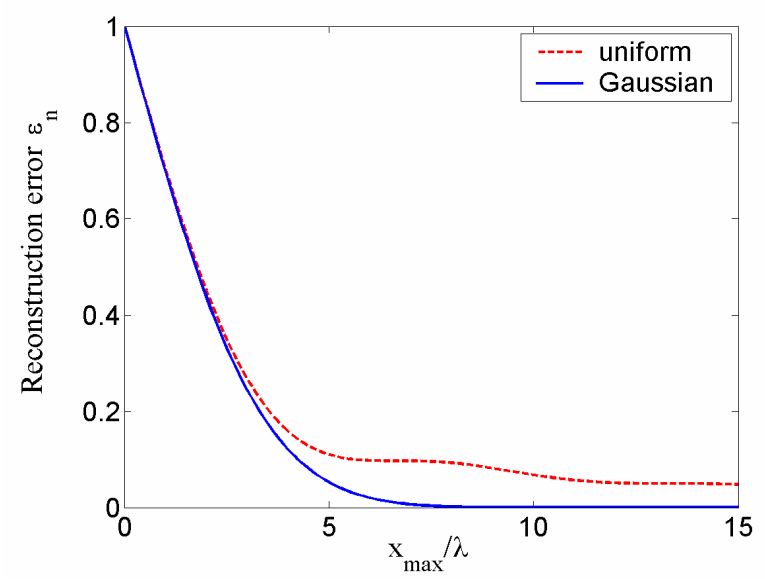

Fig 2. Normalized reconstruction error $\varepsilon_{n}$ vs. $x_{\max }$ for uniform and Gaussian PDFs; $\theta_{0}=30^{\circ}, \sigma=2.88^{\circ}\left(\Delta=10^{\circ}\right)$.

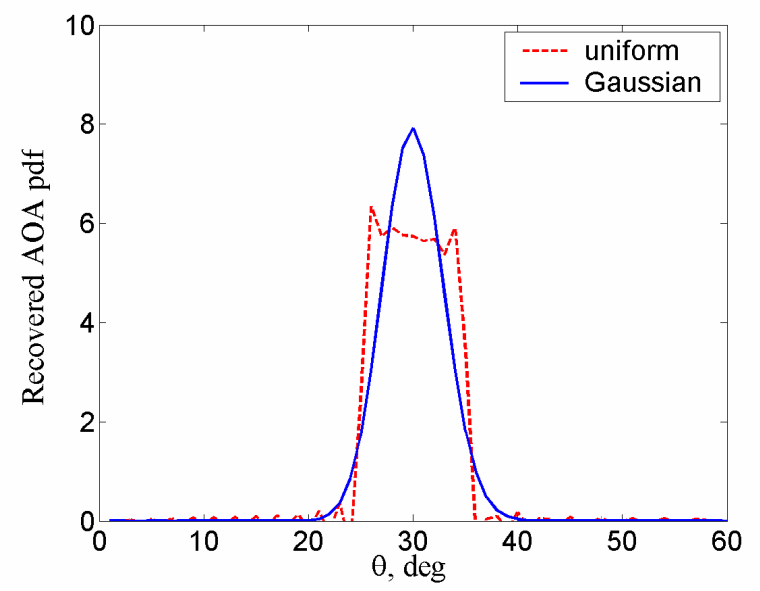

Fig 3. Reconstructed AOA PDFs for the same scenario.

\section{OPTIMIZING MIMO SYSTEM PERFORMANCE}

Using the models above, the correlation can be estimated for various scenarios. Alternatively, based on the model, the antenna design can be optimized to reduce the correlation and, hence, to improve the performance (capacity, diversity gain, BER, etc.). Traditionally, this optimization is done in terms of the minimum distance required to provide reasonably low correlation. A number of results are available for a single-cluster case with various PDFs $[2,3,5]$. Presence of multiple clusters adds 
new dimension to the optimization problem as the interaction between the clusters can be exploited in various ways to achieve low correlation for distances smaller that those of the single-cluster case.

\section{A. Minimizing the correlation}

Let us consider the scenario depicted in Fig. 4. For simplicity, two-cluster case and two-element antenna array is considered below. Low correlation can always be achieved by spacing the antennas far enough. Another way to achieve the same goal, which has not received much attention in the literature, is by rotating the antenna array (i.e. by choosing optimum $\theta$ ).

Using (20), the correlation magnitude can be expressed as (note that the correlation phase does not affect the performance in this case),

$$
|R|^{2}=w_{1}^{2}\left|R_{1}\right|^{2}+w_{2}^{2}\left|R_{2}\right|^{2}+2 w_{1} w_{2}\left|R_{1}\right|\left|R_{2}\right| \cos \left(\psi_{2}-\psi_{1}\right)
$$

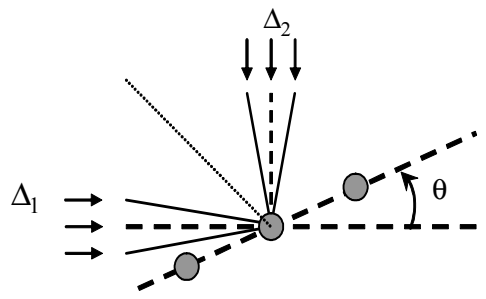

Fig 4. Optimizing the array direction for two-cluster channel.

where $\psi_{i}=k d \sin \left(\theta_{i}+\theta\right)$, and $d$ is the antenna spacing. Since $w_{1}, w_{2}$ are positive constants, $|R|$ is minimized when $\psi_{2}-\psi_{1}=(2 m+1) \pi, m$ is integer (i.e. two terms in (20) are out of phase and, if their amplitudes are close to each other, the net correlation is low). In this case,

$$
2 k d \cos (\bar{\theta}+\theta) \sin \delta=(2 m+1) \pi
$$

where $\bar{\theta}=\left(\theta_{1}+\theta_{2}\right) / 2, \quad \delta=\left(\theta_{1}-\theta_{2}\right) / 2 \quad$. If $(2 m+1) /|4 \sin \delta| \leq d / \lambda<(2 m+3) /|4 \sin \delta|$, there are $2 m+2$ solutions $\theta_{p}$,

$$
\theta_{p}=\cos ^{-1}\left[\frac{ \pm(2 p+1) \lambda}{4 d \sin \delta}\right]-\bar{\theta}, p=0,1, \ldots, m
$$

If $d / \lambda<1 /|4 \sin \delta|$, there exists no solution to (22), which means that the cluster terms are never out of phase. The correlation can still be minimized by setting $\theta=-\bar{\theta}$. All the minima above are local (except for the last case, when there is only one minimum). The global minimum can be found numerically by comparing the correlation in minima points.

In a similar way, one can find the "worst" $\theta$, for which the correlation is (locally) maximum. If $d / \lambda<1 /|2 \sin \delta| \quad, \quad \theta_{\text {worst }}=\pi / 2-\bar{\theta} \quad . \quad$ If $l /|2 \sin \delta| \leq d / \lambda<(l+1) /|2 \sin \delta|, l=1,2 \ldots$, there are $2 l$ locally "worst" angles:

$$
\theta_{\text {worst }}=\cos ^{-1}[ \pm p \lambda /(2 d \sin \delta)]-\bar{\theta}, p=1, \ldots, l
$$

As an example, consider two orthogonal clusters $\theta_{1}=0^{\circ}, \theta_{2}=90^{\circ}$. Using (23),

$$
\theta_{p}=\cos ^{-1}\left[\frac{ \pm(2 p+1) \lambda}{2 \sqrt{2} d}\right]-\frac{\pi}{4}, p=0,1, \ldots, m
$$

If $d<\sqrt{2} \lambda / 4(\approx 0.35 \lambda)$, the correlation minimum is at $\theta=-\pi / 4$. If $0.35 \lambda \leq d<\sqrt{2} \lambda / 2(\approx 0.7 \lambda)$, there are two solutions for $\theta$, and so on. Fig. 5 shows the magnitude correlation versus $\theta$ for the clusters with uniform AOA PDFs. For $d=0.5 \lambda$, there are two minima, at $\theta=0^{\circ} \quad$ and $\quad \theta=90^{\circ} \quad: \quad\left|R\left(\theta=0^{\circ}\right)\right| \approx 0.34$ and $\left|R\left(\theta=90^{\circ}\right)\right| \approx 0.30$. Hence, the optimum angle is $\theta_{\text {opt }}=90^{\circ}$. Using the same techniques, we find the worst case is $\theta_{\text {worst }}=135^{\circ}$, which maximize the correlation magnitude. For $d=3 \lambda$, there are 8 local minima. Numerical analysis shows that $\theta_{\text {opt }}=141^{\circ}$.

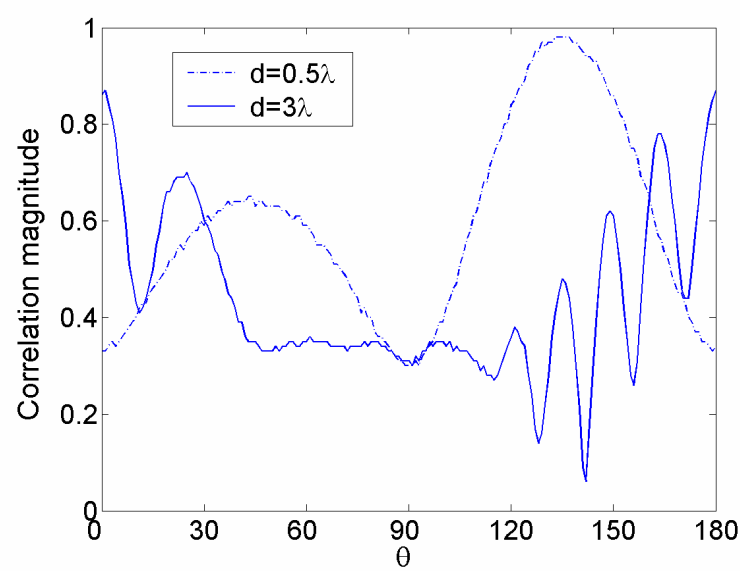

Fig 5. Magnitude correlation for two clusters with uniform AOA PDFs;

$\theta_{1}=0^{\circ}, \Delta_{1}=10^{\circ}, \theta_{2}=90^{\circ}, \Delta_{2}=20^{\circ}$

\section{B. MIMO Capacity}

Using the results above, the MIMO channel capacity can be maximized for a multiple-cluster channel. Specifically, we consider the mean (ergodic) capacity and its upper bound []. Considering 2x2 MIMO system with uncorrelated $\mathrm{Tx}$ end (i.e. all the correlation is concentrated at the Rx end), its mean capacity $\bar{C}$ and its upper bound $C_{u p}$ (in bit/s/Hz) can be expressed as [4]:

$$
\begin{gathered}
\bar{C}=\left\langle\log _{2}\left|\mathbf{I}+\frac{\rho}{2} \mathbf{H H}^{+}\right|\right\rangle \leq C_{u p} \\
C_{u p}=\log _{2}\left[1+\rho+\left(\frac{\rho}{2}\right)^{2}\left(1-|R|^{2}\right)\right]
\end{gathered}
$$

where $\mathbf{H}$ is the channel matrix. Clearly minimum correlation provides maximum $C_{u p}$. Since the mean 
capacity follows the same tendency as the upper bound, this is also true for $\bar{C}$. Fig. 6 shows mean capacity and its upper bound for a two-clustered channel (frequency-flat quasi-static Rayleigh fading) versus $\theta$. Clearly, $\vec{C}$ and $C_{u p}$ behave in a similar way, the latter being $20 \%$ less than the former. Comparing it to Fig. 5, one notices that the peaks of the correlation correspond to the dips of the capacity (both, the mean and its upper bound) and vice versa. Hence, optimization in terms of correlation and capacity are equivalent. It is interesting to note that the correlation less than approximately $0.5-0.7$ does not affect significantly the capacity and, hence, the correlation oscillation at that region do not produce corresponding oscillations in the capacity (this is especially clear for $d=3 \lambda$ ). One may also speculate that similar results should hold true for the outage capacity.

Unfortunately, these results cannot be applied directly to systems with more than 2 antennas since in that case one has to deal with the full correlation matrix rather than one parameter. Physically, this corresponds to considering the correlation of all pair of antennas, not only adjacent ones. However, approximate analysis is possible for the case of $3 \times 3$ system.

The eigenvalues $\xi$ of the correlation matrix can be found from the following:

$$
(1-\xi)^{3}-\left(2\left|R_{12}\right|^{2}+\left|R_{13}\right|^{2}\right)(1-\xi)+R_{12}^{2} R_{13}^{*}+\left(R_{12}^{*}\right)^{2} R_{13}=0
$$

where $R_{i j}$ denotes correlation between $i$ and $j(i, j=1,2,3)$ element. Noting that usually $\left|R_{12}\right|>\left|R_{13}\right|$ (i.e. distant elements are less correlated), we neglect $R_{13}$ : $R_{13}=0$. In this case, (27) allows for reasonably simple solution:

$$
\xi_{1}=1, \xi_{2}=1-\sqrt{2}\left|R_{12}\right|, \xi_{3}=1+\sqrt{2}\left|R_{12}\right|
$$

from which one notes that the approximation is valid for $\left|R_{12}\right|<1 / \sqrt{2}$ (the eigenvalues have to be positive). If this condition is satisfied, the mean capacity can be upper bounded for large SNR as:

$$
\bar{C} \leq C_{u p}=\log _{2}\left(\left(1+\frac{\rho}{3}\right)^{3}\left(1-2\left|R_{12}\right|^{2}\right)\right)
$$

The deteriorating effect of the correlation is clear. Fig. 7 shows the correlation and the mean capacity versus $\theta$ for this $3 \times 3$ system and the same as above two-cluster channel.

The approximation above (i.e. neglecting all correlation but of adjacent elements) can be extended to more than 3 antennas. In that case, however, one has to accept the following limitation: $\left|R_{12}\right|<1 / \sqrt{n-1}$, where $\mathrm{n}$ is the number of antennas. Clearly, for large $n$ the approximation is valid for small correlation (large spacing) only. It is interesting to note, however, that, as numerical analysis demonstrates, the capacity-correlation relationship still follows the same tendency as above.

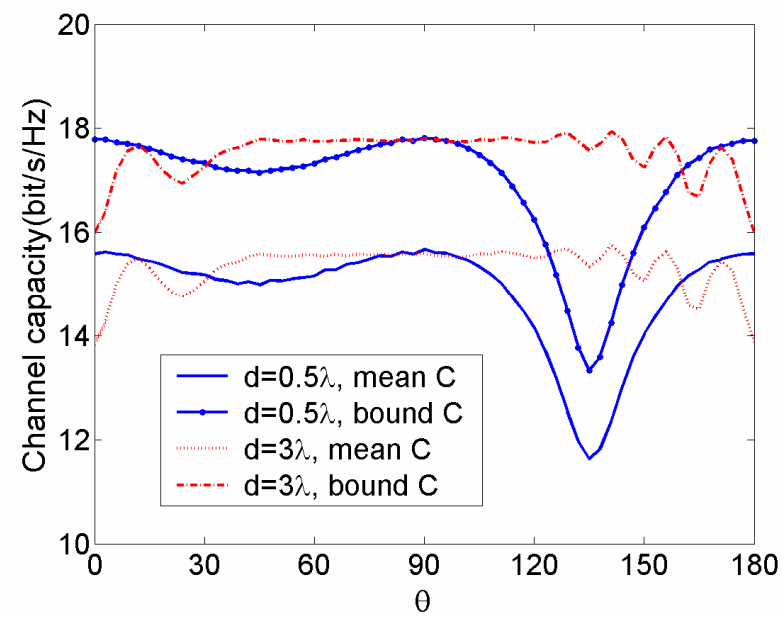

Fig 6. $2 \times 2$ MIMO mean capacity and its upper bound versus $\theta$ in a two-cluster channel with $\theta_{1}=0^{\circ}, \Delta_{1}=10^{\circ}, \theta_{2}=90^{\circ}, \Delta_{2}=20^{\circ}$ for $d=0.5 \lambda$ and $d=3 \lambda$.

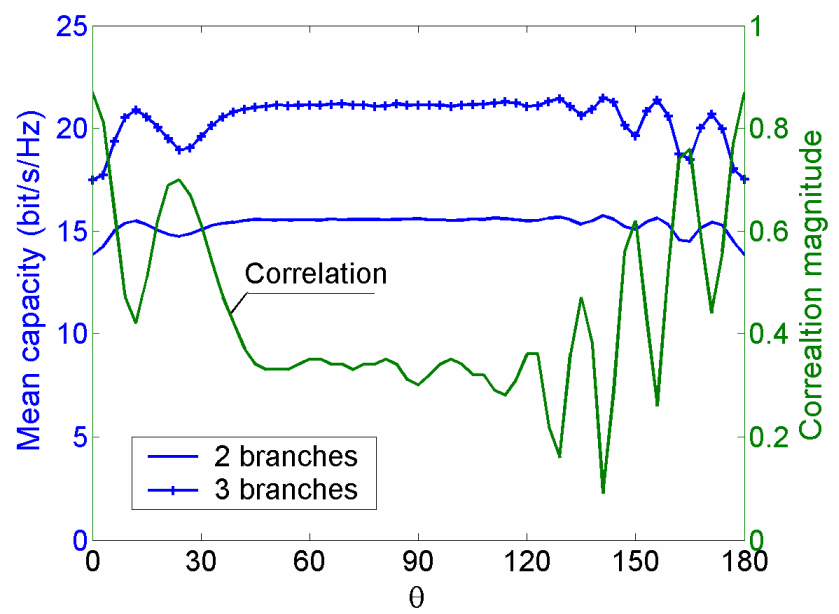

Fig 7. Mean MIMO capacity and correlation for $2 \times 2$ and $3 \times 3$ system; $\theta_{1}=0^{\circ}, \Delta_{1}=10^{\circ}, \theta_{2}=90^{\circ}, \Delta_{2}=20^{\circ}$, $d=3 \lambda$.

\section{Diversity gain}

Diversity gain can be analyzed in a similar way. Consider the outage probability (i.e. the probability that the instantaneous SNR drops below a given threshold $\gamma_{1}$ ) of a single-branch system $\operatorname{Pr}\left\{S N R<\gamma_{1}\right\}=P_{1}\left(\gamma_{1}\right)$. In a Rayleigh fading channel, $P_{1}\left(\gamma_{1}\right)=1-\exp \left(-\gamma_{1} / \Gamma\right)$, where $\Gamma$ is the average SNR [9]. For the small outage region,

$$
\gamma_{1} / \Gamma=\ln \left(1-\mathrm{P}_{1}\left(\gamma_{1}\right)\right) \approx \mathrm{P}_{1}\left(\gamma_{1}\right)
$$


For two-branch maximum ration combining (MRC) with equal average $\mathrm{SNR}$, the outage probability is [9]

$$
P_{2}\left(\gamma_{2}\right)=1-\frac{1}{2|R|}\left\{\xi_{1} \exp \left[-\frac{\gamma_{2}}{\Gamma \xi_{1}}\right]-\xi_{2} \exp \left[-\frac{\gamma_{2}}{\Gamma \xi_{2}}\right]\right\}
$$

where $R$ is the branch correlation coefficient, and $\xi_{1,2}=1 \pm|R|$. Using (31), for small outage and $R$ not too close to 1 , one obtains

$$
\gamma_{2} / \Gamma \approx\left[2\left(1-|R|^{2}\right) \mathrm{P}_{2}\left(\gamma_{2}\right)\right]^{1 / 2}
$$

Comparing $\gamma_{1}$ and $\gamma_{2}$ for the same outage probability, $\mathrm{P}_{1}\left(\gamma_{1}\right)=\mathrm{P}_{2}\left(\gamma_{2}\right)=P_{\text {out }}$, one finds the diversity gain:

$$
G \approx\left[2\left(1-|R|^{2}\right) / \mathrm{P}_{\text {out }}\right]^{1 / 2}
$$

Using the correlation analysis and expressions above, the effect of antenna geometry can be analyzed. As (33) demonstrates, the optimization in terms of diversity gain and correlation are equivalent.

Fig. 8 shows the diversity gain versus $\theta$ at $P_{\text {out }}=0.01$ for the same two-cluster channel as before. Note that the approximation (33) works well except for $\theta=135^{\circ}$ and $d=0.5 \lambda$, where $|R|=0.95$ (i.e. too close to 1$)$, where it underestimates the actual gain. The behavior of diversity gain with $R$ and $\theta$ is roughly the same as that of the MIMO capacity (i.e. peak in correlation corresponds to dip in gain; the correlation less than $0.5-0.7$ does not have significant effect, etc). Additionally, we note that the diversity gain loss for large correlation is more pronounced than that in capacity. This can be explained by the integral nature of the capacity and the local nature of the diversity gain (i.e. it is the average measure, for large time interval, and the diversity gain is local measure, for given (small) outage probability).

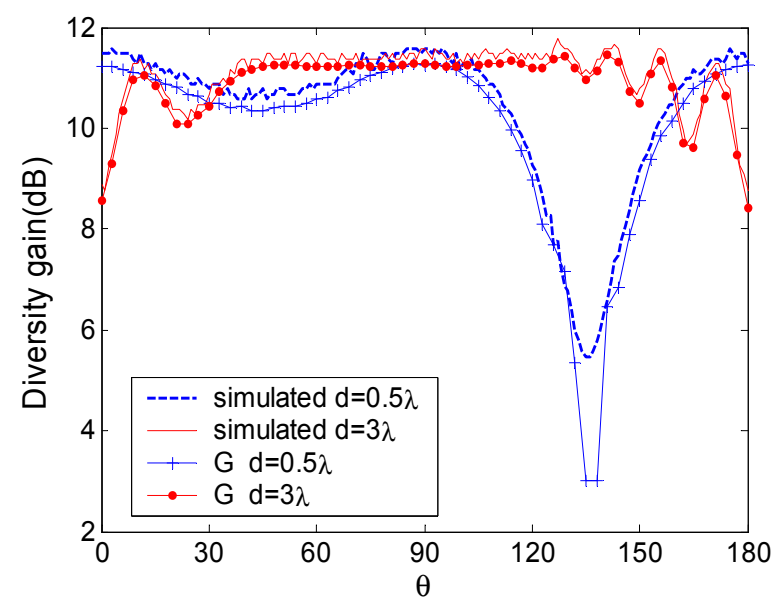

Fig. 8. Diversity gain vs. $\theta$ for 2 -branch $M R C$ in the 2-cluster channel for $P_{\text {out }}=0.01 ; \theta_{1}=0^{\circ}, \Delta_{1}=10^{\circ}$, $\theta_{2}=90^{\circ}, \Delta_{2}=20^{\circ}$.

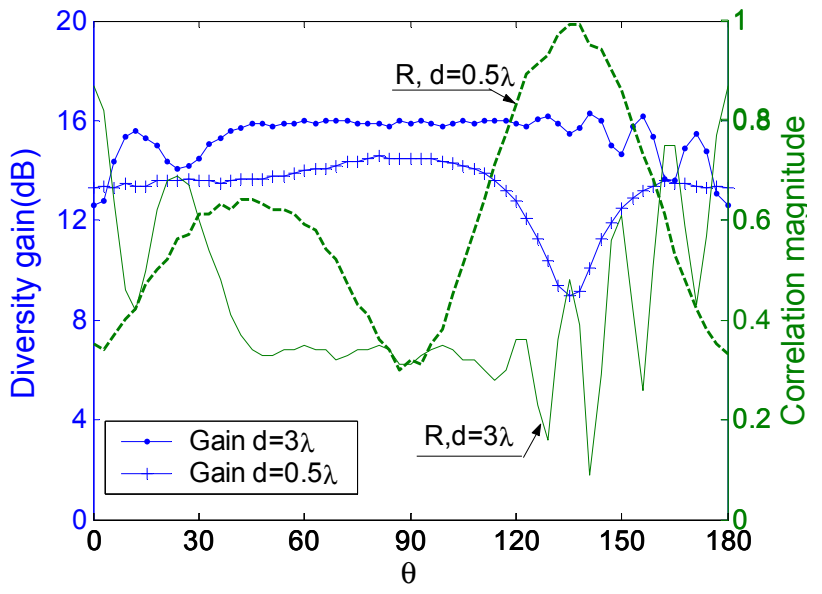

Fig. 9. Correlation and diversity gain vs. $\theta$ for 3branch MRC in the 2-cluster channel for $P_{\text {out }}=0.01$; $\theta_{1}=0^{\circ}, \Delta_{1}=10^{\circ}, \theta_{2}=90^{\circ}, \Delta_{2}=20^{\circ}$.

While the analytical analysis becomes more complex for more than two branches, the diversity gain still follows the same tendency as above, as Fig. 9 demonstrates. Note the smaller loss in the gain at $\theta=135^{\circ}$ as compared to the 2-branch system above. This is explained by the fact that while the correlation of 2 adjacent antennas ( 1 and 2, 2 and 3 ) is large, the correlation of 2 distant antennas (1 and 3 ) is lower and, hence, some diversity is still available.

\section{REFERENCES}

[1] J. Salz, J.H. Winters, "Effect of Fading Correlation on Adaptive Arrays in Digital Mobile Radio," IEEE Trans. Veh. Tech., V.43, N.4, pp. 1049-1057,Nov. 1994

[2] R. M. Buehrer, "The Impact of Angular Energy Distribution on Spatial Correlation," IEEE VTC, Vancouver, Canada, Sep. 2002, pp.1173-1177

[3] L. Schumacher et al, "From Antenna Spacing to Theoretical Capacities - Guidelines for Simulating MIMO Systems," PIMRC'02, Lisbon, Portugal, Sep. 2002, pp. 587-592.

[4] S. Loyka, G. Tsoulos, "Estimating MIMO System Performance Using the Correlation Matrix Approach," IEEE Commun. Lett., V. 6, N.1,pp. 19-21, Jan. 2002.

[5] G. Zhao, S. Loyka, "Impact of Multipath Clustering on the Performance of MIMO System", IEEE WCNC 2004, Atlanta, Georgia, USA, Mar. 2004.

[6] G. Zhao, S. Loyka, "Performance Study of MIMO System in a Clustered Multipath Channel", IEEE CCECE'04, Niagara Falls, Canada, May. 2004.

[7] R.Vaughan, "Spaced directive antennas for mobile communications by the Fourier transform method," IEEE Trans. on Ant. \& Prop., V. 48, N. 7, pp. 1025 - 1032, July 2000.

[8] G. Zhao, S. Loyka, "MIMO Capacity of Multipath Clustered Channels Using Fourier Transform Techniques," ICSP 2004, Beijing, China, Aug. 2004.

[9] W. C.Y. Lee, Mobile Communications Engineering, McGraw-Hill,1998. 